Palaeohispanica 2, (2002), pp. 159-168

\title{
EL COMPLEJO SUFIJAL -(e)sken DE LA LENGUA IBÉRICA ${ }^{1}$
}

\author{
Javier de Hoz
}

Las leyendas monetales en -esken constituyen uno de los grupos más característicos que atestiguan un elemento morfológico de la lengua ibérica. Su estudio se inicia a la vez que el estudio de la lengua ${ }^{2}$ y jugó un papel significativo en la reconstrucción de la declinación ibérica realizada por Schuchardt y que daba como resultado una declinación casi idéntica a la euscara. Curiosamente el resultado de este trabajo, hecho desde postulados totalmente distintos a los de Schuchardt y con la ventaja de conocimientos que invalidan muchas de las suposiciones del lingüista vienés, ha terminado en una interpretación como veremos muy similar a la que aquél propuso, lo cual por supuesto no quiere decir que contribuya a reforzar otras interpretaciones particulares o el cuadro general de la gramática ibérica defendido por Schuchardt.

Las bases de mi análisis son que el ibérico debe ser interpretado en la medida de lo posible por el ibérico mismo, que ese principio debe guiar la labor inicial y esencial de segmentación, y que secundariamente se debe tomar en cuenta los paralelos de inscripciones similares en culturas de similar nivel de desarrollo o que han tenido una relación directa con la ibérica. Naturalmente un paso previo es la recopilación del material que ejemplifica el problema que vamos a abordar.

\footnotetext{
${ }^{1}$ Este trabajo es en buena parte mi comunicación al X Congreso nacional de Numismática (1999): "La leyenda monetal ikalesken (MLH A.95)", la publicación de cuyas Actas parece ahora poco probable. Su versión original se realizó dentro del proyecto financiado por la DGICYT PB96-0615, y su actualización en el proyecto financiado por el Ministerio de la Ciencia BFF2000-0692-C02-01.

${ }^{2}$ Sobre los estudios del s. XIX vid. Schuchardt, H.: 1907: Die iberische, 73-5; al parecer el primero en apuntar al caracter de gentivo de nuestra secuencia y compararla con el genitivo vasco fue Longpérier en 1841. La contribución del propio Schuchardt se encontrará en op. cit., 31-9; posteriormente, sin pretender ser exhaustivo, se han ocupado del tema Beltrán, P. : 1942: Sobre, 15-8; Bähr G.: 1948: Baskisch, 113-4; Vallejo, J.: 1946: "En torno", 242-8, 2568; 1950: "Sobre ibérico" con la nota de Michelena, L.: 1950: "Sobre"; 1954: "Exploraciones", 236-7, además de la bibliografía citada infra. La idea de que la secuencia equivale a un gentivo plural se puede considerar un supuesto totalmente aceptado en la bibliografía actual que en general tiende a ver en ella un étnico, vid. por ej. Fatás, G.: 1973: La Sedetania, 49; Villaronga L.: 1979: Numismática, 125; A. Domínguez en Alfaro, C. et alii: 1997: Historia, 125, 177.
} 
El segmento -(e)sken (MLH III.1 §542, MLH I, 79s.), está atestiguado en una serie de leyendas monetales: ${ }^{3}$

[1] arsesken (A.33), auśesken (A.7), iltiŕkesken (A.19), untikesken (A.6), otobeśken (A.23), laieśken (A.13), seteisken (A.25), urkesken (A.96) e ikale(n)sken (A.95); cf. infra neronken (A.1). ${ }^{4}$

En todos estos caso la secuencia parece unirse a nombres propios, en concreto $\mathrm{NN}(\mathrm{de}) \mathrm{L}$ (ugar), como tendremos ocasión de comprobar.

Hasta la fecha no existen otros testimonios sino esas leyendas monetales repartidas desde Narbona, si neronken corresponde a la misma formación, hasta tal vez Almeria (urkesken), si las viejas localizaciones de esa ceca fuesen correctas. o más probablemente y en todo caso algún punto más septentrional del SE ibérico dentro del área de escritura meridional, que es la utilizada en las cecas A.95 y A.96, pero la mayor parte de los ejemplos proceden del NE. Casos aislados, como arsesken, permiten sin embargo atribuir el sufijo a la totalidad del territorio ibérico.

En la interpretación del sufijo hasta la fecha se han tenido en cuenta indicios muy diversos, válidos unos, arbitrarios otros. Paralelos epigráficos comprensibles más próximos son las acuñaciones ampuritanas con la leyenda "de los emporitanos", y las celtibéricas entendidas también como genitivos de plural del localicio. ${ }^{5}$ A esto se ha unido la existencia de correspondencias como:

\section{[2] auśesken / Ausetani, iltiókesken / Ilergetes, untikesken / Indigetes e Indicetani, urkesken / Urcitani,}

y algunas especulaciones vascoibéricas para atribuir al sufijo varios rasgos semánticos: indicación de étnico, de pluralidad y de pertenencia. En realidad la relación con la expresión del étnico no está nada clara, ya que existen también pares como arsesken/arse, tipo alternativo para la mayor parte de los pares ya citados puesto que existen los NNL Ausa, iltiŕke $(\mathrm{CNH} 4,1)$-atestiguada desde hace poco- ${ }^{6}$ Indica, Urci. De hecho las únicas formas en -sken relacionables con un étnico pero no con un topónimo son seteisken (A.25) junto a Sedetani y laieśken (A.13)

\footnotetext{
${ }^{3}$ Las referencias a inscripciones reenvían a MLH; las leyendas monetales, número de ceca precedido de A, al volumen I, las restantes al III. Una referencia precedida de * indica una inscripción aún no publicada en MLH pero que llevará esa identificación en el próximo suplemento; agradezco a J. Untermann el que me haya proporcionado esa información. Naturalmente en esos casos indico la bibliografía en que la inscripción ha sido dada a conocer. Las monedas todavía no conocidas cuando se publicó MLH I se citan por CNH.

${ }^{4}$ Cuadro de testimonios junto con las formas relacionadas - topónimos y étnicos- en Faust, M.: 1966: Die antiken, 34-5. Para más detalles sobre las diversas cecas vid. García-Bellido, MP. \& Blázquez, C.: 2001: Diccionario II, s. vv.

${ }^{5}$ Dadas las confusiones a las que puede dar lugar en un contexto referido al mundo antiguo el término español 'gentilicio' en su sentido de adjetivo que designa al habitante de una ciudad, derivado normalmente del nombre de ésta, utilizo en su lugar el término 'localicio' fácilmente comprensible.

${ }^{6}$ Con posterioridad a la publicación de MLH I.
} 


\section{El complejo sufijal -(e)sken de la léngua ibérica}

junto a Laietani, lo que no es indicio suficiente, de no existir otra información como la recientemente aparecida, para decidir si estamos ante un sufijo formante de étnicos, de simples localicios o indicador de funciones específicas de un topónimo; no tan probable por supuesto es que el sufijo exprese idea de pluralidad. En cuanto a la pertenencia, es razonable pero sin demostración por el momento pero es precisamente esa posibilidad unida a la final -en del sufijo o complejo sufijal lo que nos interesa en primer lugar.

La lengua ibérica tiene sin duda rasgos de tipo aglutinante; un lexema recibe sufijos morfológicamente bien definidos y aislables; esos sufijos pueden ligarse unos a otros formando auténticas cadenas. ${ }^{7}$ En el caso de los sufijos nominales las cadenas más obvias son las que se forman con los sufijos -ar-, -en y -Yi, que pueden aparecer en las combinaciones -ar-en-Yi o más simplemente -ar-Yi, -ar-en o -en-Yi. Esos sufijos o cadenas aparecen con frecuencia unidos a NNP(ersonales), es decir propios, y en cuanto a sus valores semánticos lo más claro parece ser que el sufijo -en expresa la pertenencia. ${ }^{8}$

Estos datos previos que encontramos en el ibérico mismo son nuestro necesario punto de partida y nos indican que la secuencia que estudiamos puede ser un complejo sufijal, y que en él tal vez se incluya uno de los sufijos conocidos, en concreto -en, que como en numerosos ejemplos de inscripciones de propiedad se une precisamente a nombres propios, aunque en este caso NNL.

Desde luego se ha planteado ya con anterioridad la posibilidad de que (e)sken sea un sufijo compuesto, ${ }^{9} \mathrm{y}$ de hecho el que probablemente la pertenencia sea uno de los componentes semánticos de la secuencia -(e)sken y a la vez parezca ser el significado de -en tras NNP, unido al caracter sin duda en parte aglutinante de la lengua ibérica visible en casos como los citados -ar-en o -ar-en-Yi, aconseja interpretar-(e)sken como una combinación de sufijos en la que empezaremos por aislar $-(e) s k-e n{ }^{10}$

El residuo -(e)sk- plantea, aparte la cuestión central de su significado, dos problemas, el de su auténtica forma y el de si es a su vez una combinación de sufijos o un sufijo simple. Respecto de la forma cabe la posibilidad de que ésta sea -eske- con pérdida fonética o gráfica de una $e$ en las suturas ee de las secuencias -e-eske-en, y con pérdida de la vocal final de un NL ante $e$ del sufijo cuando aquélla no era $e$. Desde este punto de vista puede ser significativo que independientemente, a partir de otros contextos, se hayan aislado en ibérico dos sufijos de forma precisamente -es y -ke.

\footnotetext{
${ }^{7}$ Sobre la gramática ibérica MLH III.1, 155-94; Michelena: 1979: "La langue"; Untermann: 1987: "La gramática"; Velaza, J.: 1996: Epigrafia; de Hoz, J.: 2001: "Hacia".

${ }^{8}$ Además de la bibliografía citada en la n. anterior vid. Michelena 1976: "Ibérico -en "; Silgo, L.: 2000: "De nuevo".

${ }^{9}$ Pero Michelena: 1985: Lengua (1955: “Cuestiones”), 362 no supone dos morfemas -(e)s y -ken; cf. MLH III.1, 184 n.173.

${ }^{10}$ Michelena, L.: 1976: "Ibérico -en “; MLH III.1, 164-5.
} 
El más visible es $k e$ (MLH III.1 § 529), identificado ya por Bähr, ${ }^{11}$ pero que a la vista de los textos greco-ibéricos plantea el problema de la posible confusión gráfica en escritura paleohispánica de hasta cuatro sufijos distintos, visibles en escritura greco-ibérica en el plomo mayor de Alcoy (G.1.1):

\section{[3] -ke: baseroke $-k$ : baśk -ge: naltinge -g: śalirg.}

De esas formas greco-ibéricas la que tiene más posibilidades de incluir un sufijo es śalirg, dada la frecuencia del término śalir en otros contextos (MLH III.1, 191-2), pero en todo caso las formas grecoibéricas subrayan la ambigüedad de las levantinas. ${ }^{12}$ De las formas terminadas en ke hay muchas que difícilmente pueden conciliarse con la hipótesis sobre -sken que aquí vamos a proponer, pero es probable que en esas formas estemos ante más de un sufijo; por ejemplo la segmenta-

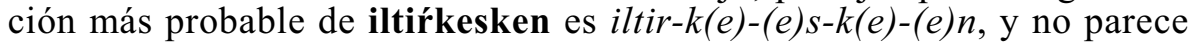
económico que ambos $\langle\mathrm{ke}\rangle$ correspondan al mismo sufijo. De hecho no podemos saber cuál era la forma fonética real del conglomerado que encontramos en las monedas, pero por el momento ninguno de los diversos -ke que encontramos en las inscripciones ibéricas se encuentra en un contexto lo suficientmente claro como para que podamos plantearnos si tiene o no la misma función que en nuestra secuencia.

El sufijo es (§ 523) está identificado también por Bähr sobre la base de un único ejemplo, ${ }^{13}$ al que luego se sumó algún otro, pero el testimonio más interesante está en un hallazgo recientísimo. Se trata de una lápida ampuritana en la que se lee: ${ }^{14}$

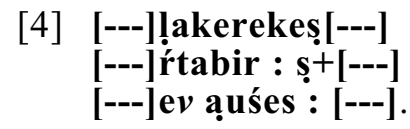

Como sostienen los editores es probable que lakerekes sea un NP, y tratándose de una inscripción sepulcral podemos esperar quizá algún otro NP - tal vez Jŕtabir - y en su caso indicaciones de parentesco, pero lo significativo es auśes que de ninguna forma se puede separar de auśesken, Ausetani y Ausa. El paralelo de las inscripciones latinas contemporáneas nos hace pensar en una indicación de origen, ${ }^{15}$ con lo que

\footnotetext{
${ }^{11}$ Bähr G.: 1948: Baskisch, 73-4, en parte apoyándose en Schuchardt.

${ }^{12}$ En ibérico existían oclusivas en posición final, pero la escritura sólo podía notar oclusivas seguidas de vocal, por lo que las finales o no eran notadas o lo eran con una grafía convencional con vocal muda.

${ }^{13}$ Bähr G.: 1948: Baskisch, 82-3.

${ }^{14}$ Aquilué, X. \& Velaza, J.: 2001: "Nueva". Corrijo ligerísimamente la lectura de los edds. y prescindo de los dos fragmentos menores que no añaden nada a la interpretación del texto.

${ }^{15}$ Así lo han visto los editores que trabajan sobre esa base en pp. 285-8, pero curiosamente en p. 284-5 piensan que auśes es el topónimo indígena.
} 


\section{El complejo sufijal -(e)sken de la léngua ibérica}

auśes significaría "de Ausa" o "ausetano" y estaría formado sobre *auśe o *auśa - no conocemos la forma indígena- con un morfo gramatical -(e)s, probablemente el mismo reiterado en -esken. Así se explican probablemente las leyendas iltirkes, ikales (A.95) y seteis (A.25) junto a las correspondientes formas en -sken; de hecho la nueva leyenda iltirke no aumentaba las probabilidades de que se tratase simplemente de casos de abreviatura, ya que como hemos visto la hipótesis del topónimo seguido del sufijo $-(e) s$ era siempre una posibilidad, ${ }^{16}$ pero ahora cabe explicar con menos dudas las formas en -es como formas plenas. ${ }^{17}$

Los otros posibles casos de sufijo -es no se encuentran en contextos que permitan añadir nada de interés, pero es interesante el caso de bastesiltiŕte[ en Liria 24 (MLH III.1 § 565) que, comparado con alauniltiŕte de Cogul (D.8.1) ${ }^{18}$ y el topónimo alaun (A.16), nos hace pensar que bastcorresponda también a un NL, con paralelos conocidos, ${ }^{19}$ seguido del mismo sufijo -es, lo que reforzaría su relación con indicaciones locales.

La presencia de un sufijo - $(e) s$ en la secuencia tiene sin embargo en contra un dato, la leyenda otobeśken (A.23), que debe corresponder a la ciudad citada en las fuentes bajo nombres diversos y con problemas de transmisión: ${ }^{20}$

[5] Otogesa (Caes. B. C. 1 61.5, 68.1, 70.4), ${ }^{21}$ 'Hтóß円бa (Ptol. Geog. 2 6.62), OTOBESANV[ (CIL II ${ }^{2}$ 14, 145 (II 3794); cf. $14,632(?)){ }^{22}$

La epigrafía latina nos da la garantía de que existió una ciudad Otobesa, a la que debe referirse la cita de Tolomeo y con menos seguridad la de César. La existencia de ese topónimo plantea la posibilidad de una formación otobeś-ken, frente a los casos hasta ahora considerados que implican -(e)sken más bien que -ken, y ello ha dado pie, desde Schu-

\footnotetext{
${ }^{16}$ Así lo defendí en el congreso arriba mencionado, antes de la aparición de la inscripción ampuritana.

${ }^{17}$ Es cierto que tenemos otro sufijo ibérico, $-k u$, que, al menos en el mosaico de Caminreal (E.7.1) parece indicar origen, pero se trata de un caso aislado y no podemos valorar en qué consistía la diferencia entre ambas formaciones.

${ }^{18}$ La defensa de esta lectura está en que proporciona dos secuencias bien conocidas, pero de hecho los signos parecen responder más bien a alaunikatiŕte.

${ }^{19}$ Aunque Basti de la Bastetania queda lejos y la ceca A.29, baśti, se diferencia en la sibilante; cf. sin embargo A.6.12, bastokiśa, aunque sólo uno de los ejemplares reproducidos en $\mathrm{CNH}, 51.99$, parece presentar esa leyenda, en los restantes, $\mathrm{CNH} 51.96-8$ y 51.100 , se lee etokiśa.

${ }^{20}$ TIR K-30: 1993, 169, carece de utilidad.

${ }^{21}$ Los manuscritos están sin duda corruptos; la forma Otogesa es la reconstrucción puramente paleográfica de varias de esas lecturas, pero también está atestiguada en algún caso; los editores han preferido tradicionalmente la lectura Octogesa que es obvia lectio facilior. Vid. Vallejo: 1946: "Sobre".

${ }^{22}$ Las menciones de otobesanos en CIL II 826 y 829, ambas en Lusitania, deben referirse a otra ciudad; el que uno de los individuos pertenezca a la tribu Galeria, como los ciudadanos de Edeta en cuyas proximidades debía estar la Otobesa que nos concierne (Alföldy en CIL II ${ }^{2}$ 14, p. 46), no es muy significativo si tenemos en cuenta que su cognomen es Vetto. Cf. Wiegels, R.: 1985: Die Tribusinschriften, 127.
} 
chardt, ${ }^{23}$ a hipótesis sobre el origen de $-s$ - en un sufijo de la propia toponimia indígena, sin que se haya prestado atención a la contradicción que implicaba considerarlo a la vez como una marca de origen. En realidad se trata de un caso aislado frente a un número muy superior de ejemplos en que estamos seguros de la existencia de un topónimos $\sin -_{-s-}$. Por ello me inclino a pensar que otobeśken ha surgido regularmente de *otobeś-esken. De hecho se trata del único caso con laieśken en que encontramos $<\dot{s}>$ en vez de $<$ s $>$, por lo que me inclino a pensar que ésta era la forma propia del sufijo, y que la $<\dot{s}>$ de otobeśken no es sino resultado de la fusión del tema del topónimo con la-s- propia del sufijo, bien -śes- > -ś-, bien -ses- >-ś-, ya que no tenemos la forma indígena del topónimo sin sufijo.

En conclusión podemos pensar como hipótesis en una combinación de elementos, $-e s=$ indicación de origen, $-e n=$ indicación de pertenecia, $-k e=$ formador de étnicos o pluralizador; las dos primeras funciones se deducen de los propios textos ibéricos, la tercera es el resto al que por exclusión llegamos en el análisis de -(e)sken una vez que hemos aclarado los otros dos elementos. auśesken sería pues, en orden inverso a la formulación ibérica "(moneda) de [pertenecia] - los/los originarios - de [origen] - Ausa". Insisto sin embargo en que esto no pasa de hipótesis.

En cuanto al caso de neronken, no está aislado puesto que existe śelonken (A.1.001), ${ }^{24}$ pero ninguna de esas formas permite deducir con seguridad cuál era el NL - la segunda carece de cualquier otra referencia y la primera plantea el problema no resuelto de la relación con el NL Narbo que ha sobrevivido en el actual Narbona- ${ }^{25}$ por lo que no sabemos si ante ke existe o no otro sufijo. Si lo hay la hipótesis más razonable con los datos actuales, aunque desde luego especulativa, sería una segmentación nero-n-ke-(e)n, con el sentido "de los en Nero", es decir una construcción locativa en vez de indicación de origen que tiene cierto apoyo en algunas cecas como bolśkan (A.40) frente a OSCA, posible indicación de "acuñado en Osca", ${ }^{26}$ si no, tendríamos un paralelo en bastike $(* \text { B. } 7.346)^{27}$ junto a bastes ya citado. Es cierto sin embargo que la ausencia de es en estos casos representa una dificultad para la hipótesis arriba planteada.

Por otro lado es curioso que, como decía al principio, por caminos distintos hayamos llegado a una propuesta que básicamente coincide con la de Schuchardt. El esquema $-s-k e-n$ al que llegaba él, dando al primer elemento el valor de indicación de origen, al segundo el de plural y al tercero el de genitivo, que sin ser idéntico en el detalle es en lo esencial

\footnotetext{
${ }^{23}$ Schuchardt, H.: 1907: Die iberische, 34-7.

${ }^{24}$ Más dudoso es si debemos incluir en el mismo grupo la ceca, catalana a juzgar por los hallazgos, A.32: ośku(Y)ken. En principio lo más probable es que sea así, y que $<\mathrm{Y}>$ se haya utilizado como representación de $/ \mathrm{n} /$ en un contexto específico. Por supuesto no puedo entrar aquí en la difícil, y en buena medida no resuelta, cuestión de ese signo; un trabajo reciente con la bibliografía anterior: Correa, J. A.: 1999: "Las nasales".

${ }^{25}$ Vid. MLH I, ad loc.

${ }^{26}$ de Hoz, J.: 1981: "El euskera", 44; 1995: "El poblamiento", 275.

${ }^{27}$ Solier, Y.: 1979: “Découverte”; Untermann: 1996: “Los plomos”, 101; 1998: “Sprache”, 38.
} 


\section{El complejo sufijal -(e)sken de la léngua ibérica}

el mismo que propongo, se basaba en la hipótesis de que el ibérico era una forma antigua de vasco, lo que le llevaba a identificar los dos últimos elementos de acuerdo con rasgos de la gramática euscara mientras que el primerto era un residuo cuyo valor se deducía del posible significado de la leyenda una vez que se habían aislado los otros dos, y se apuntalaba con explicaciones de étnicos indígenas en la versión griega o latina en la que habría quedado testimonio de $-s$. Aquí el ibérico mismo nos ha permitido proponer una segmentación de $-(e) s \mathrm{y}-(e) n \mathrm{y}$ atribuir valores a ambos elementos; ke se justifica formalmente dentro del ibérico, pero no contamos con ningún indicio de su significado, y es la leyenda en su conjunto y el significado de los otros dos elementos lo que nos permite plantear una hipótesis, como ocurría con $-s$ en el caso de Schuchardt. No se trata de mera casualidad porque Schuchardt, por muy convencido que estuviese de su hipótesis vasco-ibérica, era un lingüista demasiado fino como para desentenderse de las posibilidades reales de los textos que comentaba, pero lo cierto es que al parecer hemos llegado a una conclusión que parece consolidar una de las piezas clave en la tradición vasco-iberista. Sin embargo las cosas son bastante más complejas; es muy probable que el ibérico tuviese una marca de genitivo -en, aparentemente idéntica a la del vasco, pero en realidad para valorar ese parecido tendríamos que saber más de lo que sabemos del ibérico. En vasco parece existir una relación entre esa marca de genitivo y la marca de relativo; una relación similar en ibérico sería un dato que realmente nos obligaría a considerar la posibilidad de una relación histórica, pero por el momento no tenemos información adecuada para valorar posibles solidaridades morfológicas en el interior del ibérico. En cuanto a la hipotética marca de plural $k e$, por un lado no sabemos que se esconde en ibérico bajo $<\mathrm{ke}>$, como ya vimos puede $\mathrm{ser} / \mathrm{ke} / \mathrm{o} / \mathrm{k} /$, pero también $/ \mathrm{ge} /$ o /g/, por otro, una construcción -k-en con el significado "de los" es totalmente ajena al vasco actual, en el que la construcción es -e-(e)n. La marca $k$ sólo pluraliza el caso absoluto, que al carecer de sufijo desinencial recibe una marca especial; los restantes casos, y por supuesto el genitivo, intercalan -e- entre el singular y la desinencia como indicador de plural. Para establecer correspondencias entre vasco e ibérico -ken tenemos que partir necesariamente no del vasco tal cual lo conocemos sino de una hipótesis sobre su reconstrucción según la cual existiría una marca general de plural $k$, o en su caso $g$ relacionada con el sufijo colectivo aga, que se habría perdido en posición intervocálica; esta reconstrucción es posible aunque no segura, ${ }^{28}$ pero si tenemos que remontarnos a un estado prehistórico de la lengua será necesario preguntarse si el genitivo -en, la pieza quizá más clara de la comparación vasco-ibérica, existía en ese estadio prehistórico, y aquí se dan posibilidades, de nuevo no seguras, de que la forma actual sea una innovación a partir de una forma en -e. Es decir que en este caso la hipótesis vasco-ibérica sobre -ken en justicia tendría que situar el ibérico en relación con una forma de vasco antiguo que no había perdido todavía la $k$ de plural pero ya había introducido la $n$ de genitivo; una hipótesis que no es muy económica.

${ }^{28}$ Vid. por ej. Michelena: 1979: “La langue”, § 11. 
Pero además, si aceptamos esa hipótesis, resulta que vasco e ibérico coincidirían en un posible arcaismo, $k$, pero también en una innovación, $-n{ }^{29}$ lo que nos obligaría a hacer descender la comunidad lingüística vascoibérica a fechas no muy alejadas de la historia, lo que hace doblemente incomprensible la nula ayuda que el vasco proporciona para descifrar textos ibéricos.

Por último no podemos entresacar del conglomerado -esken los elementos que nos parezcan oportunos para establecer una comparación que pretenda llegar a una propuesta de relaciones genéticas; debemos intentar comprender lo que las fuentes nos dan tal como nos lo dan, y como subrayó en su día Bähr, ${ }^{30}$ la secuencia -esken no tiene nada de vasco.

En resumen, tenemos una segmentación e interpretación funcional de -(e)sken razonables y que a mi modo de ver son las que, en la situación actual, dan cuenta de los datos del modo más económico. Secundariamente este análisis podría tal vez mostrar coincidencias con el vasco dignas de ser archivadas a la espera de más información, pero no aporta en absoluto indicios importantes de posibles relaciones genéticas a distancia digamos media.

\section{BIBLIOGRAFÍA}

Actas I: (1976): Actas del I Coloquio sobre lenguas y culturas prerromanas de la Península Ibérica (Salamanca, 1974), Salamanca.

Actas II: (1979): Actas del II Coloquio sobre lenguas y culturas prerromanas de la Península Ibérica (Tübingen, 1976), Salamanca.

Actas III: (1985): Actas del III Coloquio sobre lenguas y culturas paleohispánicas (Lisboa, 1980), Salamanca.

Actas IV: (1987): Actas del IV Coloquio sobre lenguas y culturas paleohispánicas (Vitoria, 1985), Vitoria/Gasteiz = Studia Paleohispanica, Veleia 2-3.

Actas V: (1993): Actas del V Coloquio sobre lenguas y culturas prerromanas de la Península Ibérica (Colonia 1989) = Lengua y cultura en la Hispania prerromana, Salamanca.

Actas VI: (1995): La Hispania Prerromana = Actas del VI Coloquio sobre Lenguas y Culturas Prerromanas de la Península Ibérica (Coimbra 1994), Salamanca.

Actas VII: (1999): Pueblos, lenguas y escrituras en la Hispania prerromana. Actas del VII coloquio sobre lenguas y culturas paleohispánicas (Zaragoza 1997), F. Villar \& F. Beltrán eds., Salamanca.

\footnotetext{
${ }^{29}$ Silgo, L.: 2000: "De nuevo", 111-5, consciente de que el caracter secundario de $-n$ en el genitivo vasco sería fatal para la comparación vasco-ibérica, se ha esforzado en negar esa posibilidad, pero la cuestión sigue abierta; cf. Trask, R. L.: 1997: The History, 201.

${ }^{30}$ Bähr G.: 1948: Baskisch, 113. En vez de auśesken se esperaría algo similar a **auśetaren.
} 
Actas VIII: (2001): Religión, lengua y cultura prerromanas de Hispania = Actas del VIII Coloquio Internacional sobre Lenguas y Culturas Prerromanas de la Península Ibérica, F. Villar \& M. ${ }^{a}$ P. Alvarez eds., Salamanca.

Alfaro, C. et alii (1997): Historia monetaria de Hispania Antigua, Madrid.

Aquilué, X. \& Velaza, J. (2001): "Nueva inscripción ibérica ampuritana", Palaeohispanica 1, 277-89.

Bähr G. (1948): Baskisch und Iberisch, Bayonne (=Eusko-Jakintza 2, 320, 167-94, 381-455).

Beltrán, P. (1942): Sobre un interesante vaso escrito de San Miguel de Liria, Valencia.

Boudard, P.-A. (1859): Essai sur la numismatique ibérienne, Paris.

$\mathrm{CNH}=$ Villaronga L. (1994): Corpus Nummum.

Correa, J. A. (1999): "Las nasales en ibérico", Actas VII, 375-96.

Fatás, G. (1973): La Sedetania. Las tierras zaragozanas hasta la fundación de Caesaraugusta, Zaragoza.

Faust, M. (1966): Die antiken Einwohnernamen und Völkernamen auf -itani, -etani, Göttingen.

García-Bellido, MP. \& Blázquez, C. (2001): Diccionario de cecas y pueblos hispánicos, Madrid (= DCyP).

de Hoz, J. (1981): "El euskera y las lenguas vecinas antes de la romanización", Euskal Linguistika eta Literatura. Bide Berriak, Bilbao, 27-56.

(1995): "El poblamiento antiguo de los Pirineos desde el punto de vista lingüístico", Muntanyes i població, 271-99.

(2001): "Hacia una tipología de la lengua ibérica", ActasVIII, 335-62.

Michelena, L. (1950): "Sobre el estado actual de la cuestión del genitivo vasco en -en", Emerita 18, 221-4.

(1955): "Cuestiones relacionadas con la escritura ibérica", Emerita 23, 265-284 (= 1985: Lengua e historia, 357-370).

(1972): "Etimología y transformación", Homenaje a Antonio Tovar, 305-17 (= 1985: Lengua, 296-308).

(1976): "Ibérico -en", Actas I, 353-62 (= 1985: Lengua e historia; 379-87).

(1979): "La langue ibère", Actas II, 23-39 (= 1985: Lengua 341-56).

(1985): Lengua e historia, Madrid.

MLH = Untermann, J. (1975) ..., Monumenta .

Muntanyes i població = (1995): Muntanyes i població. El passat dels Pirineus des d'una perspectiva multidisciplinària. J. Bertranpetit i E. Vives, editors, Andorra.

Schuchardt, H. (1907): Die iberische Deklination, Sitzungsb.Akad.W., Wien.

Silgo, L. (2000): “De nuevo sobre el 'genitivo' ibérico en -en”, ELEA 3, 99-118.

Solier, Y. (1979): “Découverte d'inscriptions sur plombs en écriture ibérique dans un entrepot de Pech Maho (Sigean)", RAN 12, 55-123.

TIR K-30: (1993) = Tabula Imperii Romani. Hoja K-30 Madrid, Madrid.

Trask, R. L. (1997): The History of Basque, London \& New York. 
Untermann, J. (1973): "Le nom de Narbonne et la langue de ses habitants", Narbonne. Archéologie et histoire, 163-7. Montpellier.

(1975/1980/1990/1997): Monumenta Linguarum hispanicarum. I. Die Münzlegenden. II. Inschriften in iberischer Schrift aus Südfrankreich. III. Die iberischen Inschriften aus Spanien. IV. Die tartessischen, keltiberischen und lusitanishen Inschriften, Wiesbaden.

(1987): "La gramática de los plomos ibéricos", Actas IV, 35-56.

(1996): "Los plomos ibéricos. Estado actual de su interpretación”, Las lenguas paleohispánicas, 75-103.

(1998): "Sprache und Schrift der Iberer", Die Iberer. Informationen zur Ausstellung, Bonn.

Vallejo, J. (1946): "En torno a una vieja moneda ibérica", Emerita 14, 242-58.

(1946): "Sobre la Otogesa de César Bell. Ciu I, 61, 68 y 70", Emerita 14, 259-72.

(1950): “Exploraciones ibéricas III”, Emerita 18, 174-85.

(1950): “Sobre ibérico '-(s)ken' y '-en'”, Emerita 18, 215-20.

(1954): "Exploraciones ibéricas IV", Emerita 22, 221-57.

Velaza, J. (1996): Epigrafía y lengua ibéricas, Madrid.: 1996: Epigrafía y lengua ibéricas, Madrid.

Villaronga L. (1979): Numismática antigua de Hispania. Barcelona.

(1994): Corpus Nummum Hispaniae ante Augusti Aetatem, Madrid.

Wiegels, R. (1985): Die Tribusinschriften des römischen Hispanien, Berlin.

Javier de Hoz

Universidad Complutense de Madrid e-mail:dehoz@eucmos.sim.ucm.es 